\title{
Thermomechanical Analysis of Electrically Conductive Adhesives
}

\author{
Pavel Mach ${ }^{1 *}$, David Bušek ${ }^{1}$, Radek Polanský ${ }^{2}$ \\ ${ }^{1}$ Czech Technical University in Prague, ${ }^{2}$ University of West Bohemia \\ Email:mach@fel.cvut.cz, Phone:++420224352214
}

\begin{abstract}
Mechanical properties of isotropic electrically conductive adhesives (ICAs) are mainly influenced by their formulation and thermal processing. Thermomechanical analysis of ICAs was used with the goal to find influence of addition of two types of nanoparticles, silver nanoballs and carbon nanotubes, and two types of curing processes, on air and in vacuum, on the glass transition temperature and storage modulus of bisphenol epoxy adhesives filled with silver flakes (70 to $80 \%$ by weight).

Specimens were formed as blocks. Three groups of specimens were fabricated: specimens of adhesives used as received and cured according to the recommendation of a supplier in air; specimens of adhesives used as received and cured according to the recommendation of a supplier in vacuum, specimens of the same adhesives added with low fraction ( 0,3 to $10 \%$ by weight) of silver nanoballs, colloidal dispersion of silver, or carbon nanotubes. There were found differences in the glass transition temperatures and storage and loss moduli in dependence on the type of particles added into adhesive. It was also found that the adhesive cured in vacuum had lower thermal expansion coefficient and higher the storage and loss modulus in comparison with adhesive cured in air.
\end{abstract}

\section{Introduction}

Electrically conductive adhesives (ECAs) are materials used as an environmental friendly substitution of lead-tin solders [1]. These materials have, in comparison with solders, two significant advantages:

1. The curing temperature of adhesives is by 60 to $120^{\circ} \mathrm{C}$ lower than the soldering temperature of leadfree solders.

2. It is possible to form adhesives with anisotropic electrical conductivity (ACAs), "z" adhesives. These composites have very high electrical conductivity in " $z$ " direction that means in the direction perpendicular to a printed circuit board, and very low electrical conductivity in other directions. ACAs are mostly used for assembly of fine pitch and ultrafine pitch packages.

Lower curing temperature of adhesives in comparison with soldering temperature used in lead-free soldering process makes the use of these composite materials for assembly of heat-sensitive components or for assembly of components on heat-sensitive substrates possible. Typical application of ECAs is assembly of control circuits in a process of fabrication of LCD displays.
ACAs are used for assembly of fine pitch and ultra fine pitch packages. Adhesive assembly of these packages is more advantageous than soldering, because there in no bridging in adhesive assembly in difference with soldering.

ECAs are mostly evaluated according their electrical and mechanical properties, climatic resistivity and life time [2].

The most significant electrical property of an adhesive joint is the joint resistance. The value of the resistance of the joints formed of electrically conductive adhesive with isotropic electrical conductivity (ICAs) is 5 to $15 \mathrm{~m} \Omega$ usually. It depends on the type of adhesive, volume of adhesive in the joint and joint area. For comparison, the resistance of a soldered joint having the same joint area, the resistance is 15 to $40 \mu \Omega$. If adhesive assembly is used in equipment intended for processing of low-level signals, it is necessary to examine, instead the joint resistance, its noise and nonlinearity of a current vs. voltage characteristic, too.

Mechanical properties are as important as electrical ones. Mostly tensile strength and shear strength of adhesive joints are measured. Sometimes measurements of adhesive joints endurance against the static and dynamic mechanical load are carried out.

For examination of climatic resistivity of adhesive joints, thermal cycling $\left(-40\right.$ to $\left.80{ }^{\circ} \mathrm{C}\right)$, thermal shocks $(-80$ to $120{ }^{\circ} \mathrm{C}$ ), long time ageing at the high temperature $\left(120^{\circ} \mathrm{C}\right)$, at high humidity (near $\left.100 \% \mathrm{RH}\right)$ or at the combined climatic load $\left(80{ }^{\circ} \mathrm{C} / 80 \% \mathrm{RH}\right)$ are used usually.

The work is focused on investigation of thermomechanical parameters of ICAs [3]. Thermal expansion of samples formed of adhesives was measured as well as storage and loss moduli. Following parameters were calculated: tan delta and the glass transient temperature of adhesives. Adhesives were modified with different types of particles and influence of these particles on basic mechanical properties of adhesives was examined.

Knowledge of this information is significant for application of adhesives and completes information about basic electrical and mechanical properties of adhesives.

\section{Thermal Analysis}

Thermal analysis TA is a common name for more types of analyses [4], [5]. These analyses can be divided into following groups:

A. Methods based on energy measurement.

a. Differential (Simultaneous) thermal analysis DTA (STA).

b. Differential scanning calorimetry DSC. 
B. Methods based on measurement of mechanical properties in dependence on the temperature.

a. Thermomechanical analysis TMA.

b. Dynamic mechanical analysis DMA.

C. Other methods.

a. Thermogravimetric analysis.

b. Dilatometry.

c. Dielectric thermal analysis.

d. Thermo-optical analysis.

Following types of TA were used for our measurements: thermomechanical analysis TMA and dynamic mechanical analysis DMA.

\section{Measured Parameters of Adhesives}

Following parameters of adhesives were measured:

- Dimension changes of samples in dependence on the temperature using TMA .

- The storage modulus using DMA.

- The loss modulus using DMA.

Following parameters of adhesives were calculated:

- The glass transition temperature was found using analysis of a graph found with TMA.

- Tan delta and its dependence on the temperature.

The thermal expansion coefficient is a significant parameter, which influences reliability and life time of an adhesive joint. If a substrate, a component and adhesive will have dramatically different thermal expansion coefficients, the probability of origin of micro-cracks in adhesive will be high, especially if the joint will be treated to thermal cycling. The origin of micro-cracks limits the life-time and reliability of an adhesive joint.

The storage and loss modulus in viscoelastic solids measure the stored energy, representing the elastic portion, and the energy dissipated as heat, representing the viscous portion [6]. There is advantageous, for mechanical properties of adhesive joints, to have the storage modulus as big as possible, because this modulus represents elastic properties of adhesive joint [7].

The glass transition temperature is also an important thermomechanical parameter of adhesives. This temperature must be high to avoid problems with high changes of mechanical properties of adhesive joints near to the glass transition temperature [8]. It was found that the glass transition temperature of one sample was near to $70{ }^{\circ} \mathrm{C}$. This is too low value, because such the temperature of a conductive joint can be achieved very easy, e.g. if adhesive assembly is used for mounting of a power component.

Thermal analysis also showed that some adhesives are, if the curing time and temperature recommended by a producer are used, not fully cured. Therefore more heat cycles of the measurement were used. During the first cycle adhesives became fully cured and during the next one the thermomechanical parameters were measured.

\section{Experimental}

Samples for this work were formed as blocks having dimensions $15 \times 5 \times 1,5 \mathrm{~mm}$ in a form fabricated of silicon rubber.
There were problems joined with the block manufacturing, especially with their curing. Thickness of adhesive is usually some hundreds microns if adhesive is printed, thickness of the blocks was $1,5 \mathrm{~mm}$. Therefore voids caused by vaporization of gaseous products came into being during adhesive curing.

Samples were formed of bisphenol epoxy adhesives filled with silver flakes (70 to $80 \%$ by weight). Four formulations of basic adhesives were used: C1 (75\% silver flakes by wt.), one-component epoxy resin, C2 (70\% silver flakes by wt.), one-component epoxy resin, C3 (65\% of silver flakes by wt.), two-component epoxy resin and $\mathrm{C} 4$ (80\% silver flakes by wt.), two-component epoxy resin.

Samples manufactured of formulations $\mathrm{C} 1$ and $\mathrm{C} 2$ were modified with addition of carbon nanotubes (CNT), silver nanoballs ( $\mathrm{nAg}$ ) or colloidal dispersion of silver (kAg) - see Tab.1.

\begin{tabular}{|c|c|c|}
\hline $\begin{array}{c}\text { Sample } \\
\text { labelling }\end{array}$ & Composition & $\begin{array}{c}\text { Addition } \\
\text { (\% by wt.) }\end{array}$ \\
\hline $1 \mathrm{~A}$ & $\mathrm{C} 1+\mathrm{CNT}$ & 0,3 \\
\hline $1 \mathrm{~B}$ & $\mathrm{C} 1+\mathrm{CNT}$ & 0,67 \\
\hline $2 \mathrm{~A}$ & $\mathrm{C} 1+\mathrm{nAg}$ & 2,5 \\
\hline $3 \mathrm{~A}$ & $\mathrm{C} 1+\mathrm{kAg}$ & 2,5 \\
\hline $3 \mathrm{~B}$ & $\mathrm{C} 1+\mathrm{kAg}$ & 10 \\
\hline $4 \mathrm{~A}$ & $\mathrm{C} 2+\mathrm{CNT}$ & 0,3 \\
\hline $5 \mathrm{~A}$ & $\mathrm{C} 2+\mathrm{kAg}$ & 2,5 \\
\hline $6 \mathrm{~A}$ & $\mathrm{C} 3$ & \\
\hline $7 \mathrm{~A}$ & $\mathrm{C} 4$ & \\
\hline
\end{tabular}

Tab. 1 Samples of adhesives used for measurement,

nAg... Silver nanoballs, diameter 100-150 nm.

CNT...Carbon nanotubes, multiwalled, approx. 20-50 nm $\times$ 5-20 $\mu \mathrm{m}$.

$\mathrm{kAg}$... Colloidal dispersion of silver.

TMA measurements were carried out using two types of probes: using a macro-expanse probe and using a probe for deflection of a sample. Principles of both the probes are shown in Fig. 1.
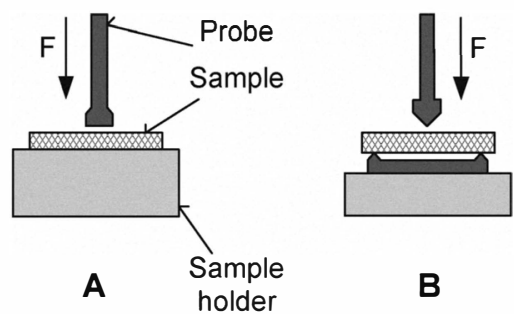

Fig. 1 Probes used for measuring using TMA A ... macroexpanse probe, $B$... probe for deflection of a sample 
DMA measurements were carried out using a probe for deflection of a sample and using a pressure probe. The probes are schematically presented in Fig. 2.

TMA and DMA were carried out using TMA Q400EM (TA Instruments) - see Fig. 3. Data are acquired automatically. Gathering, computation and graphics processing are highly sophisticated.

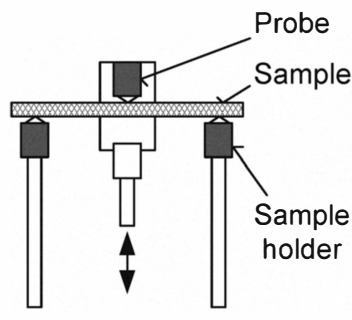

A

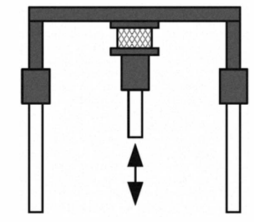

B
Fig. 2 Probes used for measuring using DMA

A ... probe for deflection of a sample, B ... pressure probe

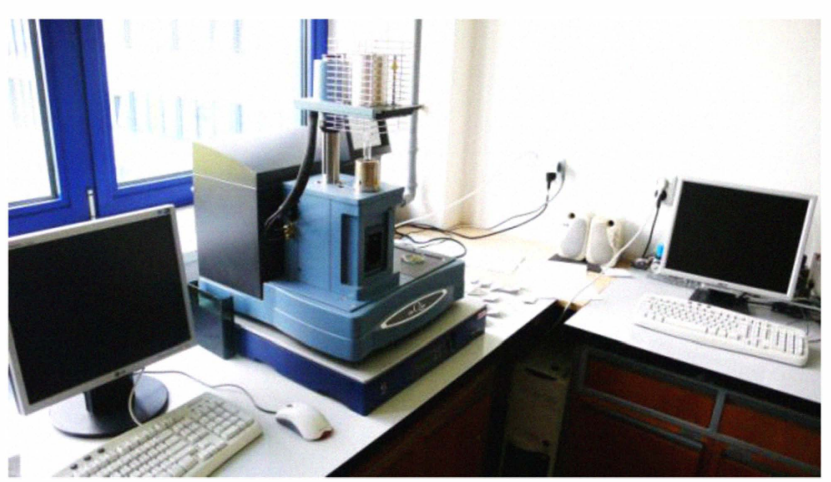

Fig. 3 Equipment used for TMA and DMA analyses

\section{Results}

First TMA was carried out. Dimension changes of samples in dependence of their temperature were found. The result measured for formulation $\mathrm{Cl}$ (bisphenol epoxy matrix with $75 \%$ silver flakes by wt.) cured in accordance with the recommendation of a supplier in air is shown in Fig. 4.

The storage modulus and loss modulus of adhesives were measured using DMA. Tan delta was calculated of these components and its dependence on the temperature was plotted. The temperature dependence of the storage modulus and the loss modulus of a sample formed of formulation $\mathrm{Cl}$ are shown in Fig. 5. The figure is completed with calculated tan delta.

Comparison of properties of samples manufactured of formulation $\mathrm{Cl}$ and cured in air (solid line) and in vacuum (dash line) is shown in Fig. 6.

Figures 4 to 6 show the results found for basic adhesive, which was not added with nanoparticles or colloidal dispersion of silver. Following figures (Fig. 7 to 9) show results, which were found for samples formed of the formulation $\mathrm{C} 3$ ( $65 \%$ of silver flakes by wt.), twocomponent epoxy resin, and of the formulation C4 (80\% silver flakes by wt.), two-component epoxy resin without addition of nanoparticles or colloidal dispersion of silver, samples 6A and 7A. These figures also show results found for samples manufactured of modified adhesives labelled C1 (75\% silver flakes by wt.), one-component epoxy resin and C2 (70\% silver flakes by wt.). Modification was carried out by addition of different types of nanoparticles or colloidal dispersion of silver. Detail composition of all adhesives under test and labelling of samples is presented in Tab. 1.

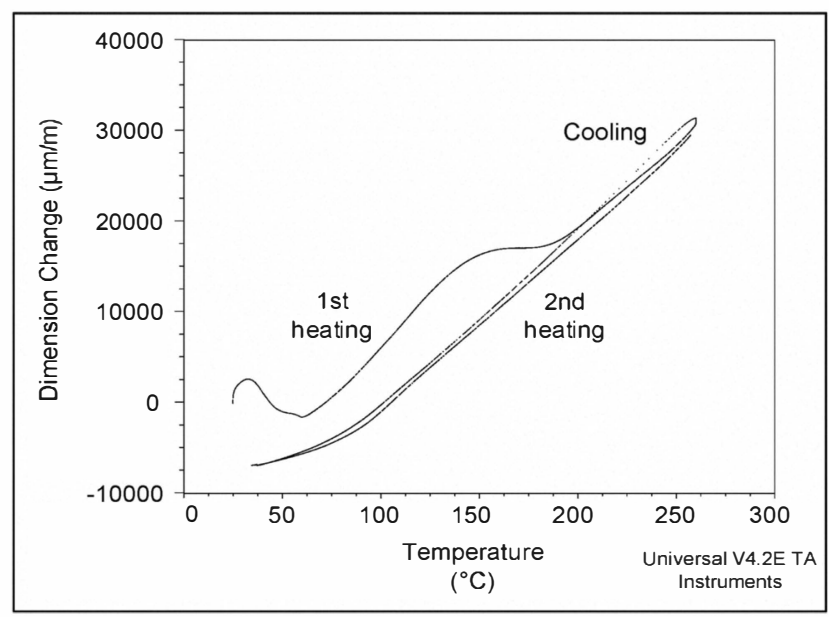

Fig. 4 TMA: formulation $\mathrm{C} 1$, cured in air

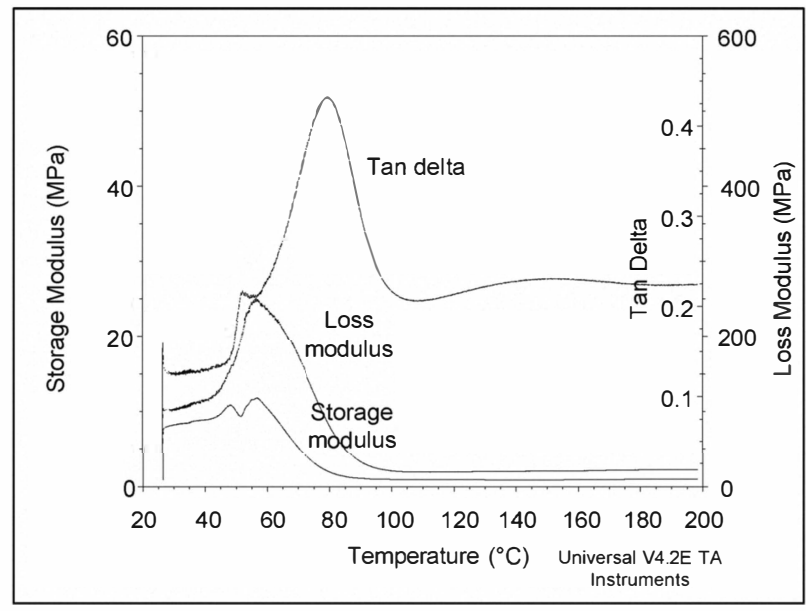

Fig. 5 DMA: formulation $\mathrm{C} 1$ cured in air, $1^{\text {st }}$ heating cycle

The storage modulus of samples measured using DMA is shown in Fig. 7. The loss modulus and tan delta of the same samples are presented in Fig. 8 and Fig. 9.

\section{Discussion of Results}

The course of thermal expansion in Fig. 4 shows that the sample manufactured of formulation $\mathrm{C} 1$ is not fully cured. The curve of the $1^{\text {st }}$ heating cycle differs significantly from the curve of cooling and the curve of the $2^{\text {nd }}$ heating cycle. 


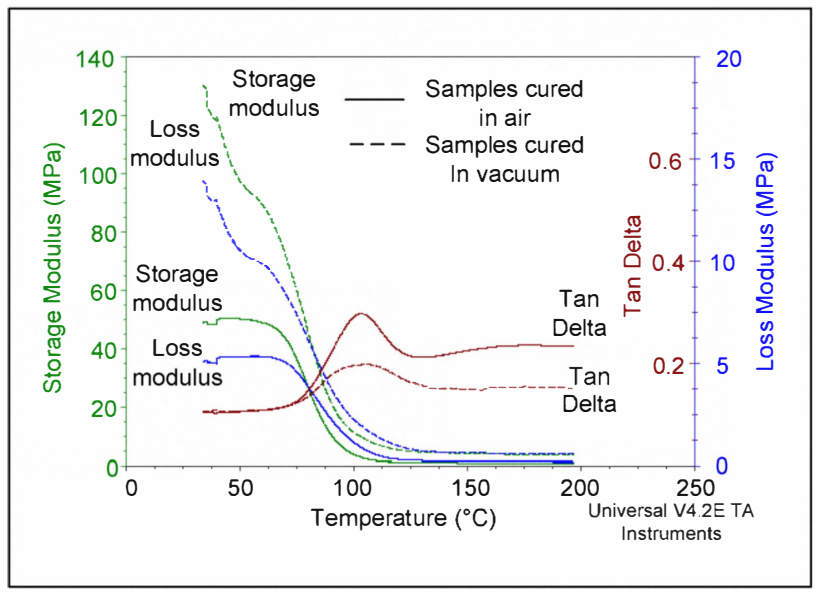

Fig. 6 DMA: storage modulus, loss modulus and tan delta of samples of formulation $\mathrm{C} 1$ cured in air and in vacuum, $2^{\text {nd }}$ heating cycle

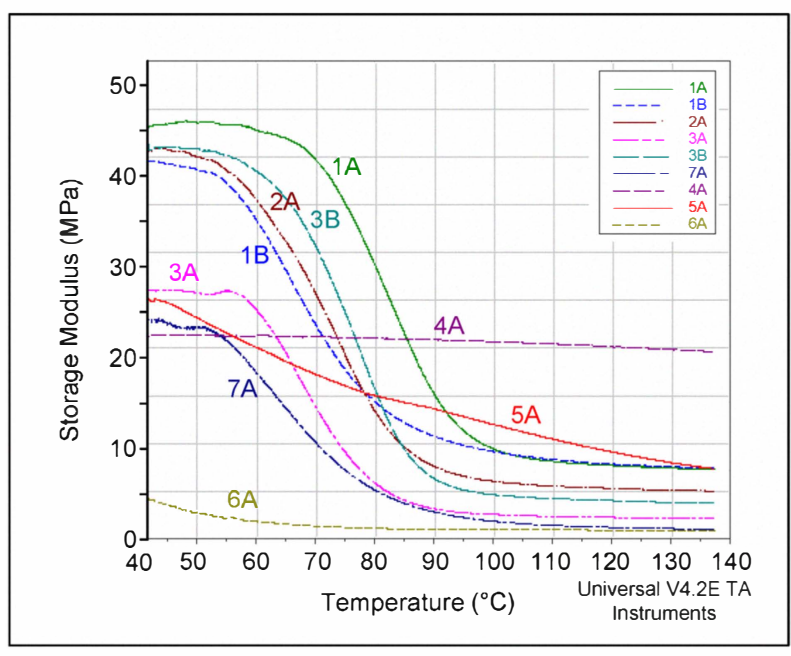

Fig. 7 DMA: storage modulus of all samples under test. Curing was carried out in air, $2^{\text {nd }}$ heating cycle

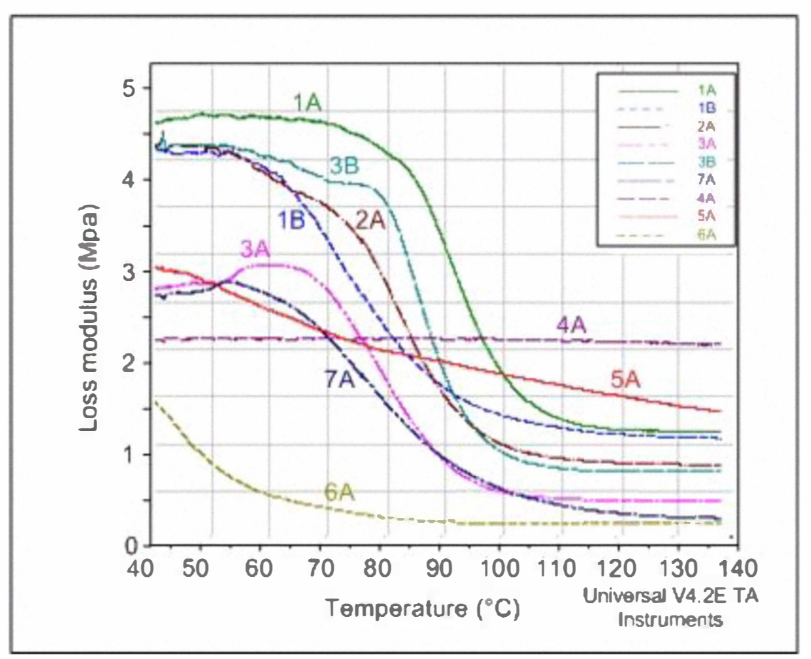

Fig. 8 DMA: loss modulus of all samples under test. Curing was carried out in air, $2^{\text {nd }}$ heating cycle

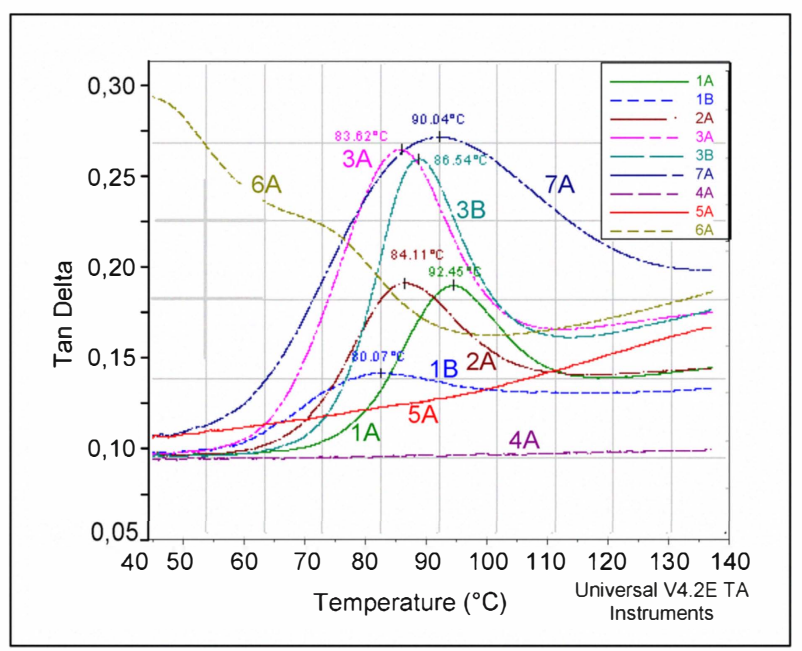

Fig. 9 DMA: tan delta of samples of formulation C1. Curing was carried out in air, $2^{\text {nd }}$ heating cycle

The fact that the sample is not fully cured causes nonlinearity in the temperature range between 60 to $200{ }^{\circ} \mathrm{C}$. In the $2^{\text {nd }}$ heating cycle the adhesive is cured already and the thermal expansion curve is in correlation with the theory.

According to the break of the thermal expansion curve for the $2^{\text {nd }}$ heating cycle the glass transition temperature was found $\left(\mathrm{Tg}=85,62{ }^{\circ} \mathrm{C}\right)[9]$, [10].

The same measurement was carried out for a sample formed of the same adhesive cured in vacuum. It was confirmed that the sample is not fully cured. The glass transition temperature was $\mathrm{Tg}=77,37^{\circ} \mathrm{C}$.

DMA was used for the measurement of the storage modulus and the loss modulus of both the samples and for calculation of tan delta [11]. Course of these moduli and tan delta for the sample cured in air during its first heating is shown in Fig. 5. Comparison with the same curves in Fig. 6 confirms the conclusion that the sample was not fully cured, that curing conditions recommended by a supporter were not optimal. Fig. 6 shows comparison of the storage and loss moduli and tan delta for samples cured under the same conditions (the temperature, the time) in air and in vacuum. It seems that curing in vacuum leads to improvement of mechanical properties and to decrease of mechanical losses. Curing in vacuum caused decrease of coefficient of thermal expansion and decrease of the glass transition temperature as well.

As for figures 7 to 9, measured values were influenced by additional curing of adhesives in the $1^{\text {st }}$ heating cycle. Therefore all figures are presented for the $2^{\text {nd }}$ heating cycle. It was found that samples $4 \mathrm{~A}, 5 \mathrm{~A}$ and $6 \mathrm{~A}$ differ significantly. The sample $6 \mathrm{~A}$ was soft, formative and it was clear that curing of this material was insufficient. Samples $4 \mathrm{~A}$ and $5 \mathrm{~A}$ do not show, in the temperature range, which was used for the test, any relaxation transitions. Measurement showed improvement of mechanical properties after the $1^{\text {st }}$ heating cycle only.

Using the storage modulus and the loss modulus tan delta was calculated. The local maxima of tan delta 
curves were used for calculation of glass transition temperatures. The highest glass transition temperature was found for the sample $1 \mathrm{~A}$, which also had low value of tan delta. Lower value of tan delta shows lower damping ability of this composite.

The sample 1A had also the highest value of the storage modulus and of the loss modulus. According to the value of the storage modulus, this adhesive had the highest mechanical strength. The course of the loss modulus showed that adhesive lost its mechanical strength at the higher temperature than other adhesives. Interesting is that comparable properties were found for the sample labelled 3B.

It was found that the best mechanical properties had the sample 1A. This sample was formed of ECA of the type $\mathrm{C} 1$ (bisphenol epoxy resin $+75 \%$ silver flake (by wt.)) added with small amount of carbon nanotubes ( $0,3 \%$ by weight). Addition of CNT caused improvement of mechanical properties of this composite. Such the influence of addition of CNT was assumed.

It is surprising that the formulation $1 \mathrm{~B}$, which is of the same type like 1A, but which has higher contents of CNT $(0,67 \%$ by weight), has worst mechanical properties than 1A. The difference is not dramatic, but it was assumed that the formulation $1 \mathrm{~B}$ will have better mechanical properties than $1 \mathrm{~A}$ due to increase of number of bonds originated by addition of CNT.

The last formulation of this type, sample $4 \mathrm{~A}$, formed of adhesive od the type C2 (bisphenol epoxy $+70 \%$ silver flakes by weight added with $0,3 \%$ CNT (by weight) has substantially worst mechanical properties in comparison with the samples $1 \mathrm{~A}$ and $1 \mathrm{~B}$. The reason could be insufficient stirring of CNT in the adhesive, therefore this experiment will be repeated.

It was also found that addition of colloidal dispersion of silver improves mechanical properties. Improvement is not so high like for the sample 1A, but it is high. The reason of this improvement is not known, it is possible that colloidal silver created with silver flakes in adhesive new bonds, which improve mechanical properties of formulation.

Comparison of values of the storage modulus, loss modulus and tan delta of adhesives having the basis $\mathrm{C} 1$ added with CNT, nAg or with colloidal dispersion of silver (samples 1A, 1B, 2A, 3A, 3B) with the storage modulus, loss modulus and tan delta of adhesive $\mathrm{C} 1$ without added particles (compare Fig. 7, Fig. 8 and Fig. 9 with Fig. 6) shows that addition of nanoparticles does not improve mechanical properties at the low temperatures. The values of these moduli or tan delta are comparable or are lower for adhesives modified with some particles. Mechanical properties of modified adhesives are mostly better for the temperatures higher than $100{ }^{\circ} \mathrm{C}$ that means over the temperatures of the glass transition.

\section{Conclusions}

Ten formulations of electrically conductive adhesives with isotropic electrical conductivity were tested. Samples of these adhesives were formed and measured using TMA and DMA. The storage modulus, loss modulus and tan delta were examined together with the thermal expansion of the samples. It was found that TMA can be successfully used for examination of a level of adhesive curing. The glass transition temperatures were found from curves of thermal expansion of samples.

The measurement of the storage moduli and the loss moduli of samples formed of different types of adhesives and the calculation of tan delta showed that these parameters are not influenced with addition of nanoparticles or colloidal dispersion of silver significantly. Improvement of these moduli and tan delta was found for modified adhesives for the temperatures over the glass transition temperature.

The measurements showed significance of thermomechanical measurements for optimization of a process of adhesive joining.

\section{Acknowledgments}

The work was carried out as a part of a project

"Diagnostics of Materials", number MSM6840770021

\section{References}

1. Luyckx, G., Dreezen, G.: Electrically Conductive Adhesives as Solder Alternative: A Feasible Chalenge. Materials for information Technology. Springer London, 2005. Pp. 363-375

2. Daoquinang Lu, Wong, C.P.: Isotropic Conductive Adhesives Filled with Low-Melting-Point Alloy Fillers, IEEE Trans. on Electronic Packagning Manuf., Vol 23, No. 3, July 2000, pp. 185-190

3. Ramachandran, V. S.: Handbook of thermal analysis of construction materials. Norwich. New York. Wiliam Andrew Publishing, 2002. pp. 680

4. Hatakeyama, T., Quinn, F. X.: Thermal analysis: Fundamentals and Applications to Polymer Science. Chichester. John Wiley and Sons. 1999. pp. 189

5. Brown, M. E.: Introduction to thermal analysis. London. Chapman and Hall. 1988. pp. 112

6. Gilham, J., Enns, J.: Trends in Polymer Science. 2, 406, 1994

7. Kaelbe, D. H.: Epoxy resins: Physical and chemical properties of cured resins. Epoxy and Technology. Marcel Dekker, pp. 327-371, 1973

8. Wunderlich, B.: Thermal Analysis of Polymeric Materials. Springer Verlag. 2005. pp. 907

9. Laredo, E. et al.: The Glass Transition in Linear Low Density Polyethylene Determined by Thermally Stimulated Depolarization Currents. Journal of Polymer Science. Part B. Polymer Physics. Vol. 34. pp. 641-648. 1996

10. Norwisz, J.: Some comments on Volds theory of DTA curve description. J. Thermal. Anal. Vol. 17, No. 2, pp. 555-557. 1979

11. Mackenzie, R. C.: Nomenclature in thermal analysis. Part IV. J. Thermal Anal. Vol. 13. No. 2. pp. 387-392. 1978 